\title{
A theoretical study of the transient and steady-state cardiorespiratory response to exercise
}

\author{
E. Magosso \& M. Ursino \\ Department of Electronics, Computer Science and Systems, \\ University of Bologna, Italy
}

\begin{abstract}
The cardiorespiratory adjustments during exercise have been analyzed through a mathematical model. It includes central chemoreceptors, responding to carbon dioxide in cerebral tissue, peripheral chemoreceptors, sensitive to arterial oxygen and carbon dioxide pressure and to arterial potassium concentration, the neurogenic mechanisms of exercise hyperpnea (including a fast and a slow dynamics), and local blood flow regulation. The model is able to simulate with good accuracy the cardiorespiratory adaptations clinically observed in response to several patterns of exercise, both as to the steady-state and dynamic conditions. In particular, the superimposition among the mechanisms included in the model reproduces the temporal heterogeneity of the ventilation response to a step work load during both the ON and OFF transitions. By using the model, the mechanisms contributing to each temporal phase have been individuated, and the functional importance of the different dynamics in the ventilatory response has been quantitatively assessed. The model may be of value to suggest possible scenarios able to interpret the complexity of ventilation control in exercise.
\end{abstract}

Keywords: mathematical models, ventilation control, exercise hyperpnea, neurogenic mechanisms, peripheral and central chemoreceptors.

\section{Introduction}

During exercise, the greatly increased metabolic demand has to be matched by an appropriate increase both of ventilation and blood flow, to provide a grater amount of oxygen $\left(\mathrm{O}_{2}\right)$ and to remove the excess of carbon dioxide $\left(\mathrm{CO}_{2}\right)$ production. In order this task is optimally performed, it is essential that several and complex series of responses are controlled and coordinated. 
Over the past decades, extensive experimental and clinical investigations have been carried out in an effort to determine the control mechanisms responsible for the observed cardiorespiratory adjustments. A generally accepted theory is the so-called neurohumoral theory $[1,2,3]$, which is based, in large part, on the dynamics of the ventilatory response to a step work load. At the exercise onset, an immediate increase in ventilation occurs, followed by a second component, which develops slower to the steady-state. The "abrupt" component is considered too rapid to be explained by humoral agents because of the delay in transport, hence it is usually attributed to neurogenic mechanisms. The second component is thought to be mediated by humoral mechanisms, such as peripheral and central chemoreceptors, which can be stimulated by substances carried in the blood, whose concentration changes during exercise. Among the proposed neurogenic mechanisms are the so-called central command (direct activation of the respiratory control centres by the locomotor stimulus), and mechano- and metabo-receptors located in exercising muscles [4]. Concerning humoral regulation, experimental studies in animals suggest that potassium [5], which is released by working muscles, increases ventilation through direct excitation of peripheral arterial chemoreceptors. On the contrary, since the arterial level of oxygen and carbon dioxide does not change significantly during exercise, these substances are commonly excluded as mediators of exercise hyperpnea.

According to the previous description, ventilation control during exercise involves a multiplicity of factors which interact in a complex way and with different dynamics. Hence, it is extremely difficult to asses the effective contribution of each involved mechanism. The scenario is further complicated by the simultaneous changes in the cardiovascular quantities (such as local blood flows), which make assessment of cause-effect relationships among variables harder and more uncertain.

Mathematical models and computer simulation techniques are of great value to investigate complex multifactorial physiological phenomena, and to recognize the functional importance of each mechanism in different conditions. Recently, we developed a mathematical model of ventilation control [6,7] (including peripheral and central chemoreceptors and central ventilatory depression), able to reproduce the ventilatory response to changes in $\mathrm{O}_{2}$ and $\mathrm{CO}_{2}$ in blood and in inspired air. In the present study, the previous model has been extended in order to include the main mechanisms involved in cardiorespiratory control during exercise: the effect of potassium on arterial chemoreceptors, the neurogenic mechanisms (including a fast and a slow dynamics) and the local blood flow regulation. The model has been used to investigate the interaction among these mechanisms and to emphasize their individual contribution both in steady-state conditions as well as in the transition from rest to exercise and vice versa.

\section{Model description}

Fig. 1 shows a schematic diagram of the main components of the model and of their reciprocal interactions. The model consists of three main compartments: the plant, the ventilation controller and the blood flow controller. The only input for 
the model is the relative intensity of aerobic exercise $(I)$, normalised with respect to individual maximum oxygen uptake. $I$ may range between 0 (corresponding to resting conditions) and 1 (corresponding to the threshold between aerobic and anaerobic exercise). The exercise stimulus $I$ directly affects tissue metabolism, arterial potassium concentration and the action of neurogenic mechanisms.

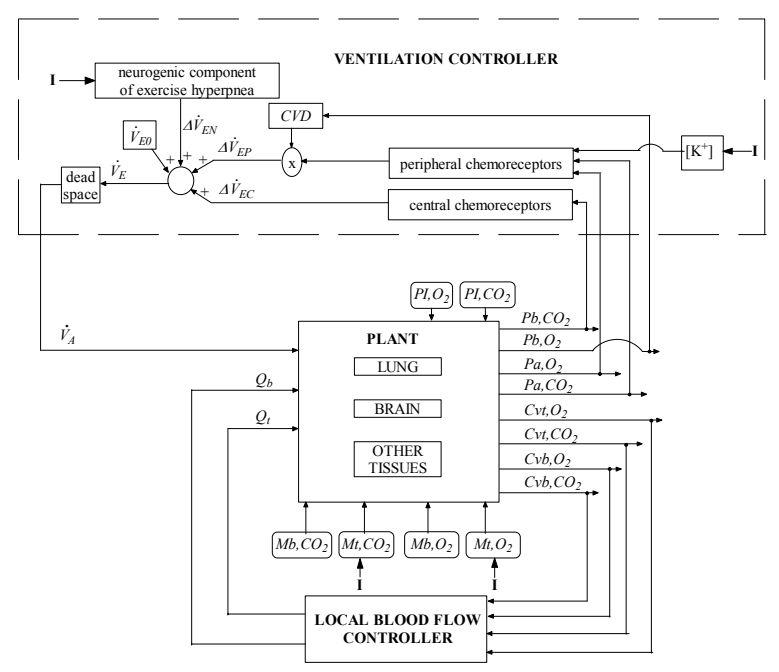

Figure 1: Schematic diagram of the model. $I$ : exercise intensity; $P$ : partial pressure of gas in inspired air $(I)$, arterial blood $(a)$ and brain tissue (b); $C v$ : gas venous concentration in venous blood leaving the brain (b) and tissues $(t) ; M: \mathrm{O}_{2}$ consumption rate and $\mathrm{CO}_{2}$ production rate in brain $(b)$ and tissues $(t) ;\left[K^{+}\right]$: arterial potassium concentration; $\dot{V}_{E 0}$ : basal value of minute ventilation; $\Delta \dot{V}_{E P}, \Delta \dot{V}_{E C}$ and $\Delta \dot{V}_{E N}$ : ventilation changes induced by peripheral $(P)$ and central $(C)$ chemoreceptors, and by neurogenic mechanisms $(N) ; \dot{V}_{A}$ : alveolar ventilation; $Q b$, and $Q t$ : blood flow to brain and to tissues.

\subsection{The plant}

The plant incorporates three compartments involved in gas $\left(\mathrm{O}_{2}\right.$ and $\left.\mathrm{CO}_{2}\right)$ storage and exchange: the lungs, brain tissue and lumped body tissues. The brain is considered separately from the other tissues since it contains the medullary chemoreceptors and the respiratory centres, hence cerebral gas pressures plays a special role in the ventilatory regulation. Equations in the plant are written by imposing a mass balance for the two chemical species in each compartment.

Oxygen consumption and $\mathrm{CO}_{2}$ production in tissues $\left(M_{t, \mathrm{O}_{2}}, M_{t, \mathrm{CO}_{2}}\right)$ depend on exercise intensity, to account for the increased metabolism of active muscles, through a static relationship and a first order low-pass dynamics. The static 
characteristic is linear for oxygen consumption whereas it is parabolic for $\mathrm{CO}_{2}$ production: indeed, carbon dioxide production increases more than oxygen uptake as exercise intensity rises [8,9]. Hence, the following equations hold:

$$
\begin{gathered}
M_{t, \mathrm{O}_{2}}=M_{t, \mathrm{O}_{2} n} \cdot\left(1+x_{\mathrm{O}_{2}}\right) \\
\frac{d x_{\mathrm{O}_{2}}}{d t}=\frac{1}{\tau_{\mathrm{O}_{2}}} \cdot\left(-x_{\mathrm{O}_{2}}+G_{\mathrm{O}_{2}} \cdot I\right) \\
M_{t, \mathrm{CO}_{2}}=M_{t, \mathrm{CO}_{2} n} \cdot\left(1+x_{\mathrm{CO}_{2}}\right) \\
\frac{d x_{\mathrm{CO}_{2}}}{d t}=\frac{1}{\tau_{\mathrm{CO}_{2}}} \cdot\left(-x_{\mathrm{CO}_{2}}+G_{\mathrm{CO}_{2}}^{\prime} \cdot I+G_{\mathrm{CO}_{2}}^{\prime \prime} \cdot I^{2}\right)
\end{gathered}
$$

where $G_{\mathrm{O}_{2}}, G_{\mathrm{CO}_{2}}^{\prime}$ and $G_{\mathrm{CO}_{2}}^{\prime \prime}$ are constant gains. $\tau_{\mathrm{O}_{2}}$ and $\tau_{\mathrm{CO}_{2}}$ represent the time constants. According to clinical findings [8,9], we assumed $\tau_{\mathrm{O}_{2}}<\tau_{\mathrm{CO}_{2}}\left(\tau_{\mathrm{O}_{2}}=40 \mathrm{~s}\right.$; $\tau_{\mathrm{CO}_{2}}=66 \mathrm{~s}$ ), that is the dynamics is slower for $\mathrm{CO}_{2}$ production than for $\mathrm{O}_{2}$ consumption.

Oxygen and carbon dioxide transport in blood is reproduced by using dissociation curves which account for the Bohr and Haldane effects, and are easily invertible.

\subsection{The ventilation controller}

The control of ventilation includes the peripheral chemoreceptors, the central chemoreceptors and the neurogenic components of exercise hyperpenea. In the model, these mechanisms interact in an additive way; hence total minute ventilation results from the following equation:

$$
\dot{V}_{E}=\dot{V}_{E 0}+\Delta \dot{V}_{E P}+\Delta \dot{V}_{E C}+\Delta \dot{V}_{E N}
$$

where $\dot{V}_{E 0}$ represents the value of minute ventilation in basal resting conditions, $\Delta \dot{V}_{E P}, \Delta \dot{V}_{E C}$ and $\Delta \dot{V}_{E N}$ denote the changes in minute ventilation induced by the activation of peripheral chemoreceptors, central chemoreceptors and neurogenic mechanisms of exercise hyperpnea, respectively.

The model provides a separate description of the afferent activity from peripheral chemoreceptors $\left(f_{p c}\right)$ and the consequent changes in ventilation ( $\left.\Delta \dot{V}_{E P}\right)$. Activity from peripheral chemoreceptors is modulated both by changes in arterial $\mathrm{O}_{2}$ and $\mathrm{CO}_{2}$ partial pressure $\left(P_{a, O_{2}}, P_{a, C O}\right)$, as well as by changes in arterial potassium concentration $\left(\left[\mathrm{K}^{+}\right]\right)$. During exercise, $\mathrm{P}_{a, \mathrm{O}_{2}}$, and $\mathrm{P}_{a, \mathrm{CO}_{2}}$ change as a consequence of alterations in tissue metabolism. Arterial potassium concentration, in the model, directly depends on exercise intensity trough a linear relationship:

$$
\left[K^{+}\right]=\left[K^{+}\right]_{n} \cdot\left(1+G_{K} \cdot I\right)
$$

The gain $G_{K}$ has been assigned so that $\left[K^{+}\right]$increases from the basal value $\left(\left[K^{+}\right]_{n}\right)$ of 4 to $6 \mathrm{mmol} / \mathrm{l}$ when aerobic exercise reaches the maximum $(I=1)[5]$. 
According to experimental data on animals [10], chemoreceptor firing depends on arterial $P_{a, O_{2}}$ through a sigmoidal relationship, whereas dependence on $P_{a, \mathrm{CO}_{2}}$ exhibits a logarithmic pattern. Chemoreceptors are sensitive to arterial potassium concentration, too [5,11]. As suggested by experimental measurements $[5,10]$, we assumed that chemoreceptor activity exhibits a multiplicative interaction between the three individual responses. Peripheral chemoreceptor firing $\left(f_{p c}\right)$ is thus described by the following equation:

$$
f_{p c}\left(P_{a, O_{2}}, P_{a, \mathrm{CO}_{2}}, K^{+}\right)=H \cdot \varphi\left(K^{+}\right) \cdot \ln \left(P_{a, \mathrm{CO}_{2}} / B_{p}\right) \cdot \frac{f_{p c \max }+f_{p c \min } \cdot \exp \left(\frac{P_{a, O_{2}}-P_{a, O_{2} c}}{k_{p c}}\right)}{1+\exp \left(\frac{P_{a, O_{2}}-P_{a, O_{2} c}}{k_{p c}}\right)}
$$

where $H$ is a constant term, necessary to adjust baseline, the quantity $\varphi$ represents the chemoreceptor response to potassium, and the meaning of all other parameters is the same as in the previous paper [6].

Since chemoreceptors perceive not only to the absolute value of $\left[K^{+}\right]$, but also its rate of change [11], the chemoreceptor response to potassium $(\varphi)$ includes a static non-linear relationship (parabolic in type) in series with a high-pass filter:

$$
\begin{gathered}
\varphi_{\text {stat }}=1+G_{K 1} \cdot\left(\left[K^{+}\right]-\left[K^{+}\right]_{n}\right)+G_{K 2} \cdot\left(\left[K^{+}\right]-\left[K^{+}\right]_{n}\right)^{2} \\
\tau_{K, p} \cdot \frac{d \varphi}{d t}=\left(\varphi_{\text {stat }}+\tau_{K, z} \cdot \frac{d \varphi_{\text {stat }}}{d t}-\varphi\right)
\end{gathered}
$$

$G_{K 1}$ and $G_{K 2}$ are constant gain factors; $\tau_{K, p}$ and $\tau_{K, z}$ are the time constants for the real pole and the real zero, with $\tau_{K, p}<\tau_{K, z}$. The time derivative of the static function $\varphi_{\text {stat }}$ is computed from the knowledge of the input $I$ waveform.

Finally, the changes in minute ventilation $\Delta \dot{V}_{E P}$ depend on $f_{p c}$ through a pure delay and a linear low-pass transfer function.

The action of central chemoreceptors on ventilation $\left(\Delta \dot{V}_{E C}\right)$ depends on brain $\mathrm{CO}_{2}$ tension through a pure delay and a linear low-pass filter. The dynamics of central chemoreceptors is slower than peripheral chemoreceptors.

The neurogenic component of exercise hyperpnea $\left(\Delta \dot{V}_{E N}\right)$ has been described in an empirical way, as a function of exercise intensity, without entering into its possible nature (i.e., central, mechano-reflex, or metabo-reflex). In particular, description of the neurogenic mechanisms includes a static non linear function (parabolic in type) and a dynamics, distinguishing between a fast and a slow component. Each of the two dynamic components is described through a pure delay and a first-order low-pass filter with different time constant. The following equations hold:

$$
\begin{gathered}
\Delta \dot{V}_{E N}=\Delta \dot{V}_{\text {fast }}+\Delta \dot{V}_{\text {slow }} \\
\tau_{f} \frac{d \Delta \dot{V}_{\text {fast }}}{d t}=\left[-\Delta \dot{V}_{\text {fast }}+\alpha \cdot \Delta \dot{V}_{s s}\left(t-D_{V}\right)\right]
\end{gathered}
$$




$$
\begin{gathered}
\tau_{s} \frac{d \Delta \dot{V}_{\text {slow }}}{d t}=\left[-\Delta \dot{V}_{\text {slow }}+(1-\alpha) \cdot \Delta \dot{V}_{s s}\left(t-D_{V}\right)\right] \\
\Delta \dot{V}_{s s}=A \cdot I+B \cdot I^{2}
\end{gathered}
$$

$\Delta \dot{V}_{\text {fast }}$ and $\Delta \dot{V}_{\text {slow }}$ represent the fast and slow component of the response, while $\Delta \dot{V}_{s s}$ is the value in steady state conditions. $\alpha$ represents the relative contribution of the fast component to the steady-state value; according to in-vivo data [8,9] its value has been set equal to $45 \%$. Finally, $A$ and $B$ are constant gains assigned to reproduce total minute ventilation measured at different exercise levels in steadystate conditions.

\subsection{The blood flow controller}

Local blood flow control, both for brain and tissue, comprehends two additive terms, which depend respectively on $\mathrm{O}_{2}$ and $\mathrm{CO}_{2}$ concentration in the venous blood leaving the compartment, through a static non-linear function and a first order dynamics. According to clinical data [4], the two local mechanisms $\left(\mathrm{O}_{2}\right.$ and $\mathrm{CO}_{2}$ ) for cerebral perfusion and the $\mathrm{CO}_{2}$ mechanism for tissue perfusion are described through static sigmoidal functions; by contrast, oxygen regulation of tissue blood flow involves an exponential static characteristic.

\section{Results}

\subsection{Model validation}

Figure 2 shows the time pattern of several ventilatory variables (total minute ventilation, alveolar $\mathrm{O}_{2}$ and $\mathrm{CO}_{2}$ pressures, oxygen consumption, carbon dioxide production and respiratory exchange ratio) measured in vivo [9] and simulated with the model in response to a 8-min step exercise. Four different exercise intensities (from mild to severe exercise) were considered. The model is able to reproduce clinical data satisfactorily, both as to the stationary and transient conditions. In particular, looking at the ventilatory response, we can distinguish two main temporal phases during the $\mathrm{ON}$ transition: a first rapid increase (lasting about half minute) which is dominated by the fast component of the neurogenic mechanisms and by the chemoreceptor high-pass response to $\mathrm{K}^{+}$; and a second slower phase gradually leading to the final value, which is mainly characterized by the slow neurogenic component and by the response of central chemoreceptor to $\mathrm{CO}_{2}$ rise. The OFF transition is characterized, after the immediate rapid decrease, by a peak, which arises from the rate dependent component of chemoreceptor response to potassium. The subsequent slow decrease to the preexercise level is due to the slow expiration of the central chemoreceptor response to $\mathrm{CO}_{2}$; this slow recover has the beneficial effect of maintaining ventilation above the resting level for few minutes after the end of exercise, allowing an increase in arterial $\mathrm{O}_{2}$ content, and the recovery of the $\mathrm{O}_{2}$ debt. 

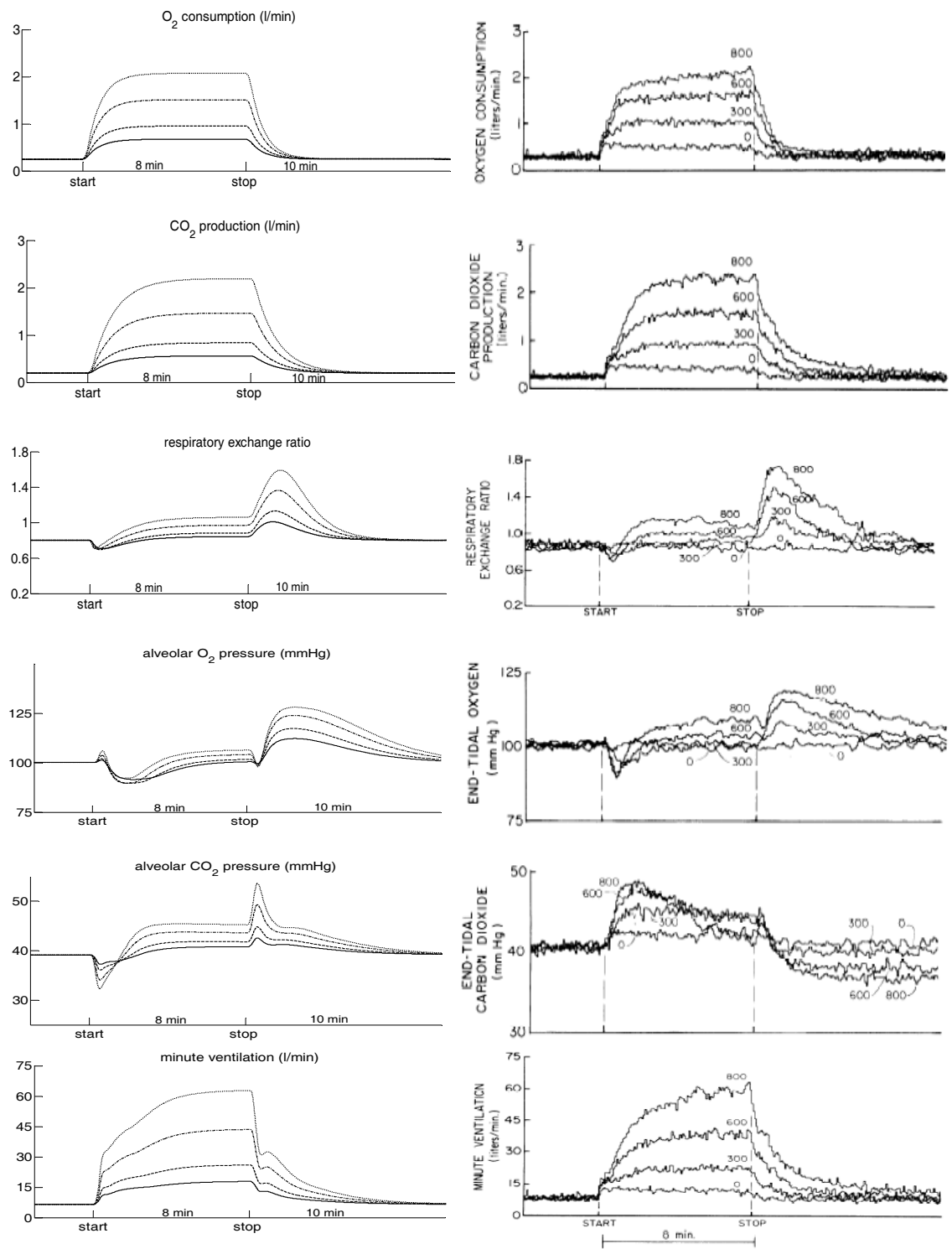

Figure 2: $\quad$ Response to a 8-min step exercise in vivo (right panels) [redrawn from Ref. 9] and simulated with the model (left panels). The transition from rest to exercise and vice-versa is accomplished in 18 seconds. Four exercise intensities are considered: $\mathrm{I}=0.15$; $\mathrm{I}=$ $0.25 ; \mathrm{I}=0.45 ; \mathrm{I}=0.65$. 


\subsection{Sensitivity analysis on regulation mechanisms}

A sensitivity analysis has been performed in order to asses the individual role of the neurogenic and humoral (that is chemoreceptor-mediated) mechanisms in exercise hyperpnea. Results are reported in Fig. 3. A 20 min moderate $(I=0.5)$ exercise, with $\mathrm{ON}$ and $\mathrm{OFF}$ transitions taking place in $1 \mathrm{~min}$, has been considered. The corresponding patterns of ventilation and arterial $\mathrm{O}_{2}$ and $\mathrm{CO}_{2}$ pressures are shown in three different conditions: with all mechanisms operative, without neurogenic mechanisms, and without chemoreflex mechanisms.

Selective elimination of neurogenic mechanisms causes a slower rise in ventilation during the $\mathrm{ON}$ phase, with a consequent greater fall in arterial $\mathrm{O}_{2}$ pressure at the beginning of exercise. However, the final value of ventilation (after the transient phase has been exhausted) differs only by $6 \mathrm{l} / \mathrm{min}$ from the value reached when all mechanisms are active. Indeed, the absence of the neurogenic mechanisms is compensated by the peripheral and central chemoreceptors, which are stimulated to a greater extent by the larger derangement of arterial gas pressures (especially carbon dioxide).

Exclusion of chemoreceptors produces a severe derangement of arterial gas content during exercise, with carbon dioxide pressure increasing up to $70 \mathrm{mmHg}$ and $\mathrm{O}_{2}$ pressure falling down to about $73 \mathrm{mmHg}$. The OFF transition in ventilation occurs much more rapidly: indeed, ventilation recovers the resting value in about 3 minutes (vs. $10 \mathrm{~min}$ ). As a consequence, at the end of exercise, a further great increase in arterial $\mathrm{CO}_{2}$ pressure occurs, while the beneficial increase in $\mathrm{O}_{2}$ content is almost completely precluded.
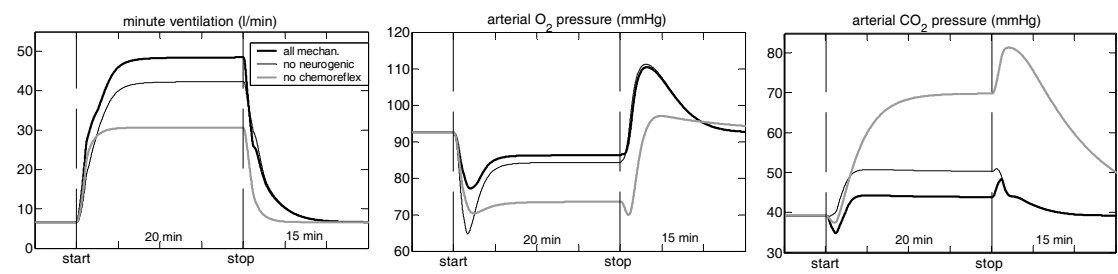

Figure 3: Response to a 20 min-step exercise simulated with the model in three different conditions: with all mechanisms operative (thick black line), without neurogenic mechanisms (thin black line), without chemoreflexes (gray line). Exercise intensity is 0.5 ; transition from rest to exercise and vice-versa is achieved in 1 minute.

\section{Discussion}

The present work represents an attempt to integrate most of the present physiological knowledge on the cardiorespiratory adjustments during exercise into a single theoretical framework. In particular, the model includes the neurogenic mechanisms, with fast and slow dynamics, the effect of alteration in humoral agents (oxygen, carbon dioxide, and potassium) on peripheral and 
central chemoreceptors, and an accurate description of local blood flow regulation in response to metabolic changes. By including the previous mechanisms the model is able to reproduce several cardiorespiratory variables in response to different patterns and levels of exercise. Such integration is of great value: indeed, despite the great amount of data gathered on this subject in the last decades, the effective contribution of each mechanism is still uncertain, and experimental results derived on individual mechanisms risk to be insufficiently understood if not related with the other results and inserted into a common theoretical setting.

The following main considerations may be drawn from simulation results. The temporal heterogeneity of the ventilation response to exercise reflects the multiplicity of the mechanisms involved, which superimpose their actions with different dynamics, ranging from few seconds to minutes. In particular, the fast neurogenic component and the high-pass response of peripheral chemoreceptors to potassium are responsible for the first rapid increase in ventilation at the beginning of exercise. The following gradual increase to the final value is mainly due to the slow neurogenic component and to the central chemoreceptors, which respond with a slower dynamics. During the OFF transition, the early abrupt decrease in ventilation arises from the fast neurogenic component (which immediately expires), whereas the subsequent slow decrease down to the resting level is accomplished by the chemoreflex responses (especially the central one), which expire more slowly.

This temporal heterogeneity has a precise functional significance. The rapid increase in ventilation at the beginning of exercise, mainly due to the neurogenic components, prevents an excessive derangement of gas content despite the concomitant changes in metabolism. In particular, it has the function of restraining the initial fall in arterial oxygen. The subsequent slower increase in ventilation up to the final value allows a consistent recovery of arterial $\mathrm{O}_{2}$ content and a moderate increase (only by few $\mathrm{mmHg}$ ) in arterial carbon dioxide.

During the OFF transition, a particular functional importance assumes the action of chemoreceptors (especially the central ones). Their activation is maintained even when exercise is finished. As a consequence, ventilation remains above the resting value for few minutes after the end of exercise, allowing arterial oxygen to increase. This excess of oxygen, at exercise termination, has the function of paying the oxygen debt which develops during exercise. In particular, the surplus of oxygen available after cessation of muscular activity is exploited for restoring the original ATP reservoir. When chemoreflexes are excluded from the regulation, ventilation recovers the preexercising level more quickly, arterial $\mathrm{O}_{2}$ does not increase at the end of exercise, and $\mathrm{O}_{2}$ debt cannot be compensated.

A further important result emerges from model simulations, which partially counteracts clinical inferences. During steady-state exercise, arterial $\mathrm{O}_{2}$ and $\mathrm{CO}_{2}$ levels exhibit only mild alterations. This usually leads to the conclusion that chemoreceptors do not play a significant role in exercise hyperpnea. In accordance with clinical findings, the model predicts only a moderate derangement of arterial gas content when all mechanisms are operative (that is in 
physiological conditions). However, in the model, chemoreceptors contribute for about $40 \%$ to the overall ventilation increase. This quite significant contribution arises from the high sensitivity of central chemoreceptors to $\mathrm{CO}_{2}$ and from the multiplicative interaction between $\mathrm{O}_{2}, \mathrm{CO}_{2}$ and $\mathrm{K}^{+}$effects on peripheral chemoreceptors. Qualitative reasoning only, which cannot account for complex and non linear interactions, may lead to a misinterpretation of the real contribution of the individual mechanisms.

In conclusion, the present model allows a rigorous assessment of the role of each regulation component in exercise hyperpnea, and it may suggest new scenarios able to interpret the complexity of the physiological reality.

\section{References}

[1] Dejours, P., Control of respiration in muscular exercise (Chapter 25). Handbook of Physiology. Respiration, sect 3, vol. 1, Am. Physiol. Soc.: Washington DC, pp. 631-648, 1964.

[2] Mines, A.H., Respiratory Physiology, Raven press: New York, pp. 133137, 1993.

[3] Ward, S.A., Control of the exercise hyperpnoea in humans: a modelling perspective. Respir. Physiol., 122 (2-3), pp. 149-166, 2000.

[4] Rowell, L.B. \& Sheperd, J.T., (eds). Handbook of Physiology, Sec.12: Exercise: Regulation and Integration of Multiple Systems, Oxford University Press: New York, pp. 333-447, 1996.

[5] Paterson, D.J., Potassium and ventilation in exercise. J. Appl. Physiol., 72(3), pp. 811-820, 1992.

[6] Ursino, M., Magosso, E., \& Avanzolini, G., An integrated model of the human ventilatory control system: the response to hypercapnia. Clin. Physiol., 21(4), pp. 447-464, 2001.

[7] Ursino, M., Magosso, E., \& Avanzolini, G., An integrated model of the human ventilatory control system: the response to hypoxia. Clin. Physiol., 21(4), pp. 465-477, 2001.

[8] Miyamoto, Y., Hiura, T., Tamura, T., Nakamura, T., Higuchi, J., Mikami, T., Dynamics of cardiac, respiratory and metabolic function in men in response to step work load. J. Appl. Physiol.: Respirat. Environ. Exercise Physiol., 52(5), pp:1198-1208, 1982.

[9] Pearce, D.H., Milhorn, H.T., Dynamic and steady-state respiratory responses to bicycle exercise. J. Appl. Physiol.: Respirat. Environ. Exercise Physiol., 42(6), pp:959-967, 1977.

[10] Fitzgerald, R.S.\& Parks, D.C., Effect of hypoxia on carotid chemoreceptor response to carbon dioxide in cats. Respir. Physiol., 12(2), pp. 218-229, 1971.

[11] Band, D.M. \& Linton, R.A.F., The effect of potassium on carotid body chemoreceptor discharge in the anaesthetized cat. J. Physiol. (London), 381, pp. 39-47, 1986. 